\title{
Relationship between Recurrent Falls and Medication Use during Acute- Care Hospitalization: A Retrospective Descriptive Study
}

\author{
Toshinori Hirai, ${ }^{*, a}$ Yutori Ishikawa, ${ }^{a}$ Yuya Kawagoe, ${ }^{a}$ Yukari Ogawa,${ }^{b}$ Ryuichi Ogawa,${ }^{c}$ and \\ Toshimasa Itoh $^{a}$ \\ ${ }^{a}$ Department of Pharmacy, Tokyo Women's Medical University Medical Center East; 2-1-10 Nishiogu, Arakawa- \\ ku, Tokyo 116-0011, Japan: ${ }^{b}$ Department of Pharmaceutical Sciences, Musashino University; 1-1-20 Shinmachi, \\ Nishitokyo, Tokyo 202-8585, Japan: and ${ }^{c}$ Department of Pharmacotherapy, Meiji Pharmaceutical University; \\ 2-522-1 Noshio, Kiyose, Tokyo 204-8588, Japan. \\ Received February 11, 2019; accepted March 27, 2019
}

\begin{abstract}
Although recurrent falls during hospitalization lead to discharge to nursing homes, their association with medications has not been comprehensively assessed. We aimed to assess risk factors for recurrent falls focusing on medications during hospitalization in an acute-care setting. This retrospective descriptive study was conducted in Tokyo Women's Medical University, Medical Center East. Patients who experienced a fall during hospitalization were included and the incidence of recurrent falls was assessed during hospitalization. Multivariate logistic regression analysis was performed to assess the relationship between recurrent falls and medications and to calculate odds ratio and $95 \%$ confidence interval. Sensitivity analysis was performed on data stratified by sex or age. This study included 124 patients with an incidence of 20 (16\%) recurrent falls. Multivariate logistic regression analysis revealed that selective serotonin reuptake inhibitors, serotonin norepinephrine reuptake inhibitors, and noradrenergic and specific serotonergic antidepressants were associated with recurrent falls (odds ratio $=\mathbf{5 . 9 8}, 95 \%$ confidence interval: $1.38-25.9, p=0.02)$. Additionally, selective serotonin reuptake inhibitors, serotonin norepinephrine reuptake inhibitors, and noradrenergic and specific serotonergic antidepressants were significant risk factors for recurrent falls in women and those aged $>80$ years. Selective serotonin reuptake inhibitors, serotonin norepinephrine reuptake inhibitors, and noradrenergic and specific serotonergic antidepressants were associated with an increased risk of recurrent falls during hospitalization in an acute-care setting. Clinicians should pay attention to patients receiving selective serotonin reuptake inhibitors, serotonin norepinephrine reuptake inhibitors, and noradrenergic and specific serotonergic antidepressants, especially women and aged $>80$ years old.
\end{abstract}

Key words recurrent fall; sex difference; elderly; selective serotonin reuptake inhibitor; serotonin norepinephrine reuptake inhibitor; noradrenergic specific serotonergic antidepressant

\section{INTRODUCTION}

A fall is recognized as a serious event that could cause fractures, a bedridden state, and prolonged hospitalization. Approximately $20 \%$ of patients with multiple falls during hospitalization were more likely to be discharged to a nursing home (not returned home). ${ }^{1)}$ Risk factors and prevention programs for falls have been reported by a number of researchers. $^{2-5)}$ For instance, medications such as benzodiazepines, antipsychotics, and selective serotonin reuptake inhibitors (SSRI) increase the risk of falls. ${ }^{2-4)}$ In addition, underweight and obesity were also associated with the increased risk of falls. ${ }^{6,7)}$

van der Velde et al. reported that withdrawal of fall riskincreasing drugs was an effective intervention to reduce the incidence of recurrent falls in geriatric outpatients. ${ }^{8)}$ The greatest risk reduction was observed when cardiovascular medications were withdrawn, whereas withdrawal of psychotropic drugs was not significantly associated with a lower recurrent fall risk. ${ }^{8)}$ In addition, multi-component intervention prevented approximately $20 \%$ of falls. ${ }^{9)}$ However, most study locations were not in acute-care hospitals but in nursing homes and community dwellings.

Clinical reports on risk factors of recurrent falls are scarce compared with those on initial falls. ${ }^{10)}$ Although loop diuretics and central nervous system-active medications were as- sociated with recurrent falls, ${ }^{11,12)}$ the relationships between recurrent falls during hospitalization and medications have not been comprehensively analyzed. According to previous reports showing an association between falls and medications, reconciliation of medications is considered essential to prevent recurrent falls during hospitalization as one of the multicomponent preventive interventions. Therefore, nurses assess patient's risk of falls, and then preventive interventions are performed in our hospital. Nevertheless, recurrent falls remain one of the most concerned clinical events in our hospital.

The aim of this study was to identify the risk factors for recurrent falls and patients who are highly sensitive to risk factors, focusing on medications during hospitalization in an acute-care setting.

\section{METHOD}

Study Design and Patients This retrospective descriptive study was conducted at Tokyo Women's Medical University, Medical Center East. All patients who experienced a fall during hospitalization between April 2016 and May 2017 were included. Patients aged $<20$ years were excluded from this study. This study protocol was approved by the institutional review board (\#4881).

Data Collection We collected patients' background 
information from their electronic medical record. The collected information was demographics (age, sex, height, body weight, and body mass index (BMI)), length of hospitalization, days from hospital admission to the initial fall, clock time of the initial fall, days from the initial fall to the first recurrence, activity of daily living (bed rest and complete bed rest), comorbidities (cardiovascular disease, cerebrovascular disease, orthopedic disease, infectious disease, gastrointestinal disease, nephrological and urological disease, dementia, and other diseases), laboratory data (hemoglobin, aspartate aminotransferase (AST), alanine aminotransferase (ALT), total bilirubin, serum creatinine, and estimated glomerular filtration rate (eGFR)), and medications of interest (hypnotics and anxiolytic drugs, antipsychotics, antidepressants, antiepileptics, antidementia medications, antihypertensives, diuretics, organic nitrates, benign prostatic hyperplasia medications, and overactive bladder medications). The percentage of those aged $<60$ years was calculated. The eGFR was calculated using a prediction equation recommended by Japanese Society of Nephrology. ${ }^{13)}$ The classifications of the retrieved medications were defined as follows, hypnotic and anxiolytic drugs were benzodiazepines (BZD), Z-drugs (i.e., zopiclone and zolpidem), and ramelteon, or suvorexant; antidepressants were serotonin selective reuptake inhibitors (SSRI), serotonin norepinephrine reuptake inhibitors (SNRI), noradrenergic and specific serotonergic antidepressants (NaSSa), and tricyclic and tetracyclic antidepressants; antidementia medications were cholinesterase (ChE) inhibitors, except for distigmine, and $N$-methyl-D-aspartate (NMDA) antagonists; antihypertensives were adrenergic $\beta$-receptor blockers, renin-angiotensin system inhibitors, and calcium channel blockers; medications for benign prostatic hyperplasia were adrenergic $\alpha_{1}$-receptor blockers, $5 \alpha$-reductase inhibitors (5ARI), and phosphodiesterase type 5 (PDE5) inhibitors; and medications for overactive bladder were distigmine and adrenergic $\beta_{3}$-receptor agonists. Clock time of the initial fall during the hospitalization was stratified as nighttime (0:00-5:59), morning (6:00-11:59), afternoon (12:00-17:59), and evening (18:00-23:59). We followed-up patients' medical records until they were discharged from our hospital or experienced a recurrent fall for the first time.

Outcome and Definitions Our study outcome was a recurrent fall during the hospital stay. A fall was defined as an incident in which a patient intentionally came to rest upon the ground or surface lower than his or her original station other than as a violent blow, loss of consciousness, onset of paralysis as in stroke, or an epileptic seizure. ${ }^{14)}$ Fall were categorized into two groups as once-only fall or recurrent fall. During the study period, patients who had fallen at least once were allocated to the once-only fall group while those who had fallen twice or more were allocated to recurrent fall. Presence of falls was evaluated according to reports by floor nurses on the medical records. Floor nurses were instructed to submit a report regarding a fall event immediately. Evaluation day was defined as the day when the recurrent fall was detected. If there were multiple recurrences, the first recurrence was used for our analysis. Patients without any recurrent fall were evaluated based on the data at discharge.

Statistical Analysis According to a previous report, ${ }^{8)}$ we assumed that $30 \%$ of patients who experienced accidental falls after the hospital admission had repeated additional falls during the hospital stay. To detect risk factors for recurrent fall using multiple logistic regression analysis with alpha and beta errors of $<5$ and $<20 \%$, respectively, 10 recurrent fallers are required per independent variable. Thus, we assumed at least 134 fallers must be included in this study to detect four independent variables with sufficient statistical power. Continuous data were expressed as mean and standard deviation (S.D.) or median and interquartile range, as appropriate. Categorical data were expressed as the number of patients and percentages. Student's $t$-test or Mann-Whitney's $U$-test was used to compare continuous variables between recurrent fall and once-only fall groups, as appropriate. Heterogeneity for categorical data was tested using Chi-square test or Fisher's exact test. We performed multivariate logistic regression analysis to calculate odds ratios (OR) and the $95 \%$ confidence intervals $(95 \% \mathrm{CI})$ of clinical predictors for recurrent falls, including medications prescribed at evaluation day (i.e., the day of the first recurrence or the day of discharge). We could not perform the time-to-event analysis because prescriptions are changed frequently in a short period in acute-care settings. The dependent variable was a recurrent fall and independent variables were demographic data, activity of daily living, the presence of comorbidity, clinical laboratory data, medications of interest, and clock time of the initial fall. Since there have been reports that initial falls occurring at 4:00-11:59 were associated with recurrent falls, ${ }^{15}$ ) we simplified the clock time as morning or not for our data analysis. Prior to performing multivariate logistic analysis, we retrieved potential independent variables with a cut-off $p$-value of 0.20 from univariate analyses. If there was a multicollinearity between independent variables, we selected one of them based on clinical relevance. Using the stepwise forward selection method, the final logistic regression model was built according to Akaike's Information Criterion. The stepwise backward selection method was used to certify the robustness of the final model. Moreover, we investigated whether there was any interaction between medications. In addition, to investigate the high-risk population, we performed sensitivity analyses stratified by sex and age ( $\leq 80$ or $>80$ years) because women and older adults are at high risk for falls. ${ }^{16)}$ Since patients aged $>80$ years had a high risk of falling irrespective of sex, ${ }^{17)}$ we used $>80$ years as a cut-off point. Interaction between medications and sex or age was investigated where possible. The statistical analyses were performed using JMP Pro 13 (SAS Institute, Cary, NC, U.S.A.). A $p$-value of less than 0.05 was considered statistically significant.

\section{RESULTS}

Figure 1 shows the flowchart of our patient selection. Among 13650 inpatients, 126 patients $(0.92 \%)$ experienced falls during our study period. After excluding two patients aged $<20$ years, the remaining 124 patients were included in our data analysis. Twenty of the patients $(16 \%)$ experienced recurrent falls after the initial fall during the hospitalization, and 5 out of the 20 patients repeated one or more recurrences. The patient background of our study population is shown in Table 1. Mean age of our study population was $74.8 \pm 10.4$ years, and seven $(6 \%)$ of the patients were aged $<60$ years. More than half of the patients were men. Body weight and BMI were lower in patients with recurrent falls than in those 


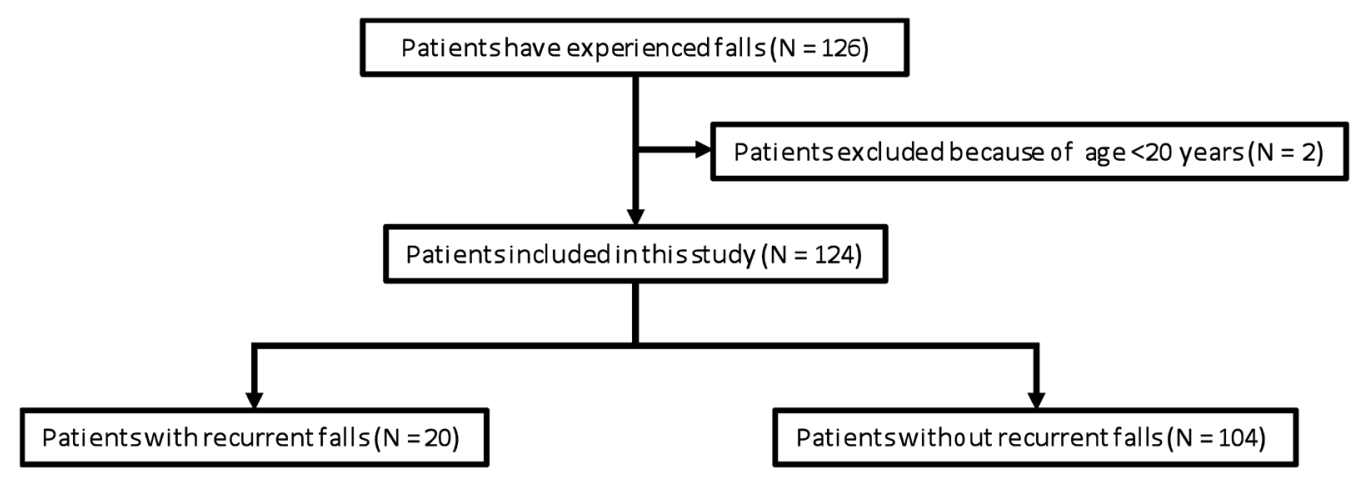

Fig. 1. Flowchart of Patient Selection

without recurrent falls although the differences were not significant. SSRI, SNRI, and NaSSa were prescribed more to patients with recurrent falls than to those without. The median days [interquartile range] from hospitalization to the initial fall was 9 [2-20]d in all patients, and it was comparable between the recurrent fall and once-only fall groups (14 [4-33]d versus $9[2-17] \mathrm{d}, p=0.13)$. The percentage of morning falls for the initial event was higher in the recurrent fall group than in the once-only fall group. The correlation between clock time of the initial and recurrent falls in the recurrent fall group is shown in Fig. 2, and nighttime fallers repeated a fall in the same time zone $(r=0.46)$.

According to the predefined strategy of our statistical analysis, BMI, presence of cardiovascular disease, use of SSRI, SNRI, or NaSSa, and clock time of the initial fall in the morning were incorporated into the multivariate logistic regression analysis (Table 2). The effects of antipsychotics and antiepileptic could not be evaluated because no patients with recurrent falls had these variables. There was multicollinearity between body weight and BMI, and BMI was used for this analysis. Using a stepwise forward selection method, use of SSRI, SNRI, or NaSSa (OR $=5.98,95 \%$ CI: $1.38-25.9$, $p=0.02)$ and clock time of the initial fall in the morning $(\mathrm{OR}=3.21,95 \% \mathrm{CI}: 1.07-9.62, p=0.04)$ were detected as statistically significant dependent variable for recurrent falls. A stepwise backward selection method confirmed this result. There was no significant interaction between use of SSRI, SNRI, and NaSSa and other medications.

The interaction between use of SSRI, SNRI, and NaSSa and sex $(p=0.31)$ was not statistically significant. However, a sensitivity analysis stratified by sex showed that the use of SSRI, SNRI, and NaSSa (OR $=15.5,95 \% \mathrm{CI}: 1.80-133, p=0.01)$ and clock time of the initial fall in the morning $(\mathrm{OR}=9.14,95 \%$ CI: $1.17-71.1, p=0.04)$ were associated with recurrent falls in female but not in male patients (Table 3). Similarly, the interaction between use of SSRI, SNRI, and NaSSa and age $(p=0.29)$ was not statistically significant while the significant risk of use of SSRI, SNRI, or NaSSa for recurrent falls was attenuated in a $\leq 80$-year-old sub-population (Table 4).

\section{DISCUSSION}

The present retrospective descriptive study demonstrated that SSRI, SNRI, and NaSSa use and clock time of the initial fall in the morning (6:00-11:59) were significant risk factors for recurrent falls during hospitalization in an acute-care hospital. Female or $>80$-year-old patients might be a high-risk population of recurrent falls due to SSRI, SNRI, and NaSSa.

Although underweight and obesity were associated with the development of fall, ${ }^{6,7)}$ BMI was comparable between recurrent and a once-only fall in our patients. Although, BMI was categorized as underweight (BMI $<18.5 \mathrm{~kg} / \mathrm{m}^{2}$ ) or obesity $\left(\right.$ BMI $>25 \mathrm{~kg} / \mathrm{m}^{2}$ ), underweight and obesity were not risk factors for recurrent falls in our study population (data not shown). As mentioned above, patients aged $>20$ years were included in this study. However, the number of patients aged $<60$ years was limited and when we excluded these patients, the results were comparable. Moreover, central nervous system medications were generally recognized as one of the risk factors for falls. ${ }^{2-4)}$ However, central nervous system medications except for SSRI, SNRI, and NaSSa were not detected as a risk factor for recurrent falls in our study. Since SSRI, SNRI, and NaSSa have relatively longer half-lives than other central nervous system medications (e.g., benzodiazepines and so-called "Z-drugs"), the carryover effect might keep patients at high risk of falls throughout the hospital stay. As indicated in previous studies performed at nursing homes or residential care settings, ${ }^{2,18)}$ SSRI, SNRI, and NaSSa are risk factors for recurrent falls. Thus, it is essential to identify patients administered SSRI, SNRI, and NaSSa irrespective of medical care setting.

Clock time of recurrent falls seemed to correlate slightly to that of initial falls by visual inspection (Fig. 2). To the best of our knowledge, there is no report on the chronological consistency between initial falls and subsequent falls. Although patients who experienced initial falls in the daytime could experience recurrences at a wide range in the daytime, the initial nighttime fallers trend to repeat falls in the nighttime. Therefore, assessing nighttime behavior (e.g., nocturnal awakenings and nocturia) may contribute to reducing recurrent falls in patients who previously experienced falls in the nighttime. Furthermore, 55\% of the first recurrences occurred within 1 week after the initial falls occurred in our study population (data not shown). This is compatible with a report that recurrent falls were observed in approximately $60 \%$ of patients within $3 \mathrm{~d}$ of hospitalization. ${ }^{6}$ ) Thus, an emphasis should be placed on careful monitoring of fallers by clinical staff, especially for the first week after the latest fall to reduce recurrences effectively.

We could not evaluate the relationships between drug exposure and the risk of recurrent falls because our retrospective study was small-scale. A previous report suggested that SSRIs, even at low doses, are associated with falls irrespective of concomitant use of hypnotics or sedatives. ${ }^{2}$ 
Table 1. Patient Background

\begin{tabular}{|c|c|c|c|}
\hline Characteristics & Recurrent fall $(n=20)$ & Once-only fall $(n=104)$ & $p$-Value \\
\hline Age, years & $73.4 \pm 11.6$ & $75.0 \pm 10.2$ & 0.53 \\
\hline Male, $n(\%)$ & $13(65)$ & $57(55)$ & 0.40 \\
\hline Height, cm & $159.5 \pm 6.7$ & $158.2 \pm 9.9$ & 0.59 \\
\hline Body weight, $\mathrm{kg}$ & $48.8 \pm 10.7$ & $54.4 \pm 13.6$ & 0.10 \\
\hline Body mass index, $\mathrm{kg} / \mathrm{m}^{2}$ & $19.2 \pm 3.4$ & $21.6 \pm 4.9$ & 0.05 \\
\hline Length of hospitalization, days & $35[19-51]^{a)}$ & $26[14-51]^{a)}$ & 0.61 \\
\hline Days from hospital admission to initial fall, days & $14[4-33]^{a}$ & $9[2-17]^{a)}$ & 0.13 \\
\hline Days from initial fall to recurrent fall, days & $6[0-22]^{a)}$ & - & NA \\
\hline \multicolumn{4}{|l|}{ Activity of daily living } \\
\hline Bed rest, $n(\%)$ & $6(30)$ & $18(17)$ & 0.21 \\
\hline Complete bed rest, $n(\%)$ & $0(0)$ & $4(4)$ & NA \\
\hline \multicolumn{4}{|l|}{ Comorbidity } \\
\hline Cardiovascular disease, $n(\%)$ & $5(25)$ & $12(12)$ & 0.13 \\
\hline Cerebrovascular disease, $n(\%)$ & $3(15)$ & $13(13)$ & 0.76 \\
\hline Orthopedic disease, $n(\%)$ & $1(5)$ & $14(14)$ & 0.24 \\
\hline Infectious disease, $n(\%)$ & $1(5)$ & $10(10)$ & 0.48 \\
\hline Gastrointestinal disease, $n(\%)$ & $2(10)$ & $7(7)$ & 0.62 \\
\hline Nephrological and urological disease, $n(\%)$ & $2(10)$ & $7(7)$ & 0.62 \\
\hline Dementia, $n(\%)$ & $1(5)$ & $5(5)$ & 0.97 \\
\hline Other diseases, $n(\%)$ & $6(30)$ & $41(39)$ & 0.43 \\
\hline \multicolumn{4}{|l|}{ Clinical laboratory data } \\
\hline Hemoglobin, g/dL & $11.3 \pm 2.5$ & $11.3 \pm 1.9$ & 0.96 \\
\hline $\mathrm{AST}, \mathrm{IU} / \mathrm{L}$ & $21.0[16.0-26.5]^{a)}$ & $21.0[15.5-31.5]^{a)}$ & 0.83 \\
\hline $\mathrm{ALT}, \mathrm{IU} / \mathrm{L}$ & $15.0[9.0-26.0]^{a)}$ & $17.0[10.0-25.0]^{a)}$ & 0.89 \\
\hline Total bilirubin, $\mathrm{mg} / \mathrm{dL}$ & $0.7[0.5-1.1]^{a}$ & $0.7[0.5-1.0]^{a)}$ & 0.92 \\
\hline Serum creatinine, $\mathrm{mg} / \mathrm{dL}$ & $0.9[0.7-2.6]^{a)}$ & $0.9[0.7-1.3]^{a)}$ & 0.64 \\
\hline $\mathrm{eGFR}^{b)}, \mathrm{mL} / \mathrm{min} / 1.73 \mathrm{~m}^{2}$ & $56.8[22.4-79.0]^{a)}$ & $55.4[42.3-77.3]^{a)}$ & 0.64 \\
\hline \multicolumn{4}{|l|}{ Medications of interest } \\
\hline Benzodiazepines $^{c)}, n(\%)$ & $2(10)$ & $19(18)$ & 0.37 \\
\hline Zopiclone or zolpidem, $n(\%)$ & $4(20)$ & $12(12)$ & 0.30 \\
\hline Ramelteon or suvorexant, $n(\%)$ & $1(5)$ & $7(7)$ & 0.77 \\
\hline Antipsychotics, $n(\%)$ & $0(0)$ & $8(8)$ & NA \\
\hline SSRI, SNRI, or NaSSa, $n(\%)$ & $4(20)$ & $5(5)$ & 0.02 \\
\hline Other antidepressants, $n(\%)$ & $2(10)$ & $5(5)$ & 0.36 \\
\hline Antiepileptics, $n(\%)$ & $0(0)$ & $8(8)$ & NA \\
\hline ChE inhibitors or NMDA antagonists, $n(\%)$ & $1(5)$ & $5(5)$ & 0.97 \\
\hline$\beta$-Blockers, $n(\%)$ & $2(10)$ & $13(13)$ & 0.75 \\
\hline Renin-angiotensin system inhibitors, $n(\%)$ & $6(30)$ & $37(36)$ & 0.63 \\
\hline Calcium channel blockers, $n(\%)$ & $5(25)$ & $38(37)$ & 0.32 \\
\hline Diuretics, $n(\%)$ & $3(15)$ & $29(28)$ & 0.23 \\
\hline Organic nitrates, $n(\%)$ & $1(5)$ & $6(6)$ & 0.89 \\
\hline$\alpha_{1}$-Blockers, 5ARI, or PDE5 inhibitors, $n(\%)$ & $2(10)$ & $12(12)$ & 0.84 \\
\hline Distigmine or $\beta_{3}$-agonists, $n(\%)$ & $1(5)$ & $3(3)$ & 0.89 \\
\hline Number of medications of interest, per patient & $2[0-3]^{a)}$ & $2[1-3]^{a)}$ & 0.30 \\
\hline Clock time of initial fall & & & 0.21 \\
\hline Nighttime $(0: 00-5: 59), n(\%)$ & $3(15)$ & $31(30)$ & \\
\hline Morning (6:00-11:59), $n(\%)$ & $7(35)$ & $17(16)$ & \\
\hline Afternoon (12:00-17:59), $n(\%)$ & $5(25)$ & $31(30)$ & \\
\hline Evening (18:00-23:59), $n(\%)$ & $5(25)$ & $25(24)$ & \\
\hline
\end{tabular}

Data are means + S.D. unless otherwise specified. ${ }^{a)}$ Median [interquartile range]. ${ }^{b)}$ eGFR was calculated using prediction equation. ${ }^{13)} c$ ) Benzodiazepines include hypnotics and anxiolytic drugs (ex; etizolam, brotizolam, and triazolam). Abbreviations: ACE, angiotensin-converting enzyme; ALT, alanine aminotransferase; AST, aspartate transaminase; ChE, cholinesterase; eGFR, estimated glomerular filtration rate; NA; not applicable, NaSSa; noradrenergic and specific serotonergic antidepressant; NMDA, $N$-methyl-Daspartate; PDE5, phosphodiesterase type 5; SNRI, serotonin norepinephrine reuptake inhibitor; SSRI, selective serotonin reuptake inhibitor; 5 ARI, $5 \alpha$-reductase inhibitors.

Although information on the fall risks of SNRI and NaSSa is limited, ${ }^{19-21)}$ several studies demonstrated that the fall risks of SNRI and NaSSa are comparable to those of SSRIs. ${ }^{20,21)}$ The potential mechanism of recurrent falls is drowsiness and muscle weakness; however, evidence of this mechanism is lacking. Furthermore, SSRI, SNRI, and NaSSa increase the incidence of fracture and falls. ${ }^{22-24)}$ Current use of SSRI and SNRI is associated with an increased risk of fractures, ${ }^{25}$ ) and patients with a history of falls particularly have a higher risk of fractures than those with no history do. ${ }^{25)}$ SSRIs and mirtazapine decrease bone mineral density and increase the incidence of fractures, which is associated with their serotonin 
Table 2. Result of Multivariate Logistic Regression Analysis for Recurrent Falls

\begin{tabular}{|c|c|c|c|c|c|c|}
\hline \multirow{2}{*}{ Variables } & \multicolumn{3}{|c|}{ Univariate } & \multicolumn{3}{|c|}{ Multivariate } \\
\hline & OR & $95 \% \mathrm{CI}$ & $p$-Value & OR & $95 \% \mathrm{CI}$ & $p$-Value \\
\hline BMI $\left(\mathrm{kg} / \mathrm{m}^{2}\right)$ & 0.89 & $0.78-1.01$ & 0.06 & & & \\
\hline Cardiovascular disease & 2.56 & $0.79-8.30$ & 0.13 & & & \\
\hline SSRI, SNRI, or NaSSa & 4.95 & $1.20-20.4$ & 0.04 & 5.98 & $1.38-25.9$ & 0.02 \\
\hline Initial fall in the morning $(6: 00-11: 59)$ & 2.76 & $0.96-7.92$ & 0.07 & 3.21 & $1.07-9.62$ & 0.04 \\
\hline
\end{tabular}

Only variables with $p$-value $<0.20$ in univariate logistic regression analysis. Abbreviations: BMI, body mass index; CI, confidence interval; OR, odds ratio; SSRI, selective serotonin reuptake inhibitor; SNRI, serotonin norepinephrine reuptake inhibitor; NaSSa, noradrenergic and specific serotonergic antidepressant.

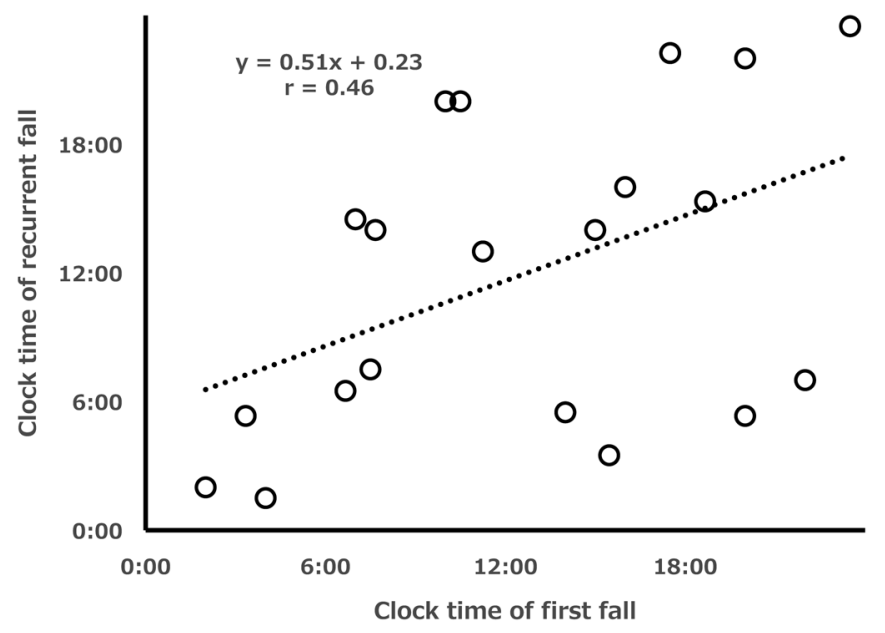

Fig. 2. Correlation between Clock Time of the Initial Falls and Recurrent Falls

transporter inhibitory effect. ${ }^{24)}$ Therefore, clinicians should continue prescribing the minimum required dose of SSRI, SNRI, and NaSSa to avoid recurrent falls and fractures. Additional studies are needed to determine whether individual medications such as fluvoxamine, a CYP inhibitor, increase the risk of recurrent fall.

The morning (6:00-11:59) fallers showed an increased risk of recurrent falls in our study population. Since the inpatient activities are relatively high during this period because of transfers to laboratory facilities, rehabilitation activities, and walking around the hospital. In addition to the highly active state, the pharmacological effect of medicines administered at bedtime (e.g., hypnotics) could persist to the early morning and promote the risk for recurrent fall of SSRI, SNRI, and NaSSa. Furthermore, a systematic review demonstrated that at least one fall caused the development of the fear of falling, resulting in a decline in physical and mental performance. ${ }^{26)}$ Collectively, these findings led us to speculate that patients who fell in the morning tended to be active but physically limited and had reduced mental performance. Therefore, we consider that paying extra attention to these patients and trying to recover their physical and mental performance could contribute to preventing subsequent falls.

For female sex, SSRI, SNRI, and NaSSa could be a significant risk factor for recurrent falls but there was no interaction between them. Women showed a better response to antidepressant treatment than men did in several studies. ${ }^{27)}$ Focusing on pharmacokinetics, sex difference was observed with several antidepressants. For instance, clearance of duloxetine was reduced in female patients compared to male patients. ${ }^{28,29)}$
Table 3. Multivariate Logistic Regression Analysis for Recurrent Falls Stratified by Sex

\begin{tabular}{lccc}
\hline \hline Variables & OR & $95 \%$ CI & $p$-Value \\
\hline Female (Number = 54) & & & \\
$\quad$ SSRI, SNRI, or NaSSa & 15.5 & $1.80-133$ & 0.01 \\
$\quad$ Initial fall in the morning (6:00-11:59) & 9.14 & $1.17-71.1$ & 0.04 \\
Male (Number = 70) & & & \\
$\quad$ SSRI, SNRI, or NaSSa & 2.69 & $0.22-33.3$ & 0.44 \\
$\quad$ Initial fall in the morning (6:00-11:59) & 1.79 & $0.46-6.98$ & 0.40 \\
\hline
\end{tabular}

Abbreviations: CI, confidence interval; OR, odds ratio; SSRI, selective serotonin reuptake inhibitor; SNRI, serotonin norepinephrine reuptake inhibitor; NaSSa, noradrenergic and specific serotonergic antidepressant.

Table 4. Multivariate Logistic Regression Analysis for Recurrent Falls Stratified by Age

\begin{tabular}{lrrr}
\hline \hline Variables & OR & $95 \%$ CI & $p$-Value \\
\hline$>$ 80 years (Number =37) & & & \\
$\quad$ SSRI, SNRI, or NaSSa & 17.2 & $1.10-267$ & 0.04 \\
$\quad$ Initial fall in the morning (6:00-11:59) & 3.37 & $0.45-25.0$ & 0.23 \\
$\leq$ & & & \\
$\quad$ S0 years (Number = 87) & 3.75 & $0.59-23.9$ & 0.16 \\
$\quad$ Initial fall in the morning (6:00-11:59) & 3.33 & $0.83-13.4$ & 0.09 \\
\hline
\end{tabular}

Abbreviations: CI, confidence interval; OR, odds ratio; SSRI, selective serotonin reuptake inhibitor; SNRI, serotonin norepinephrine reuptake inhibitor; NaSSa, noradrenergic and specific serotonergic antidepressant.

Moreover, the women were older than the men in our study population (data not shown), and exposure to duloxetine may be relatively high in women. However, we could not confirm that women had a higher exposure to duloxetine than men did because we did not measure blood concentration of duloxetine. In addition, female sex itself was a risk factor for falls. ${ }^{16}$ Therefore, clinicians should monitor recurrent falls closely in women administered SSRI, SNRI, and NaSSa treatments.

The recurrent fall risk of SSRI, SNRI, and NaSSa was emphasized in elderly ( $\geq 80$ years) sub-population. In the contrast, the risk of fall is high in 70-80-year-old women compared to $\leq 70$-year-old men. ${ }^{17}$ ) Although we attempted a further analysis, it was difficult because the 70-80-year-old patients were fewer than those in other studies. Thus, it does not necessarily mean that this sub-population (70-80-year-old) was not at risk for recurrent fall. Since drug metabolism by CYP enzymes decreases with age, ${ }^{30)}$ hepatically metabolized medications such as SSRI, SNRI, and NaSSa shows a relatively long duration of action in older patients. Moreover, the muscle mass was lower in the elderly than it was in young adults in a previous study, ${ }^{31)}$ Thus, the elderly ( $>80$ years) 
showed increased incidences of recurrent falls possibly caused by an interaction of pharmacokinetic changes and physiological strength.

This study has a few limitations. First, the study design was retrospective descriptive study with a limited sample size because electrical medical records were not introduced in our hospital before April 2016. Therefore, our study was biased to focus on recently introduced medications, change of medications, and pathophysiological changes that could contribute to the occurrence of a fall. Furthermore, the prevalence of recurrent falls in this study was lower than initially expected. There is a possibility of false positive error based on the number of independent variables. In addition, the dose-response relationship and stratification of SSRI, SNRI, and NaSSa could not be assessed because of the power of this study. Second, we did not account for some well-known risk factors for falls (e.g., the presence of vertigo, visual impairment, fear of falling, low muscle mass, physical status, and mental status) because unbiased validated information was not retrievable from our electrical medical recording system. Moreover, although a significant relationship has been reported between cognitive function and falls, ${ }^{32)}$ we could not collect Mini-Mental State Examination score, which is an index of cognitive dysfunction. Therefore, we were not able to assess the impact of cognitive dysfunction on recurrent falls in our study. Thus, the possibility of confounding factors must be considered. Third, we could not perform causality assessments between recurrent fall and medications using Naranjo's Algorithm because we could not measure drug concentration and assess the dose modification after a fall. Fourth, caution should be exercised in considering the generalizability of these results. For instance, health care systems (ex; hospitalization) in western countries differ from those in Japan. Finally, further studies would be required to examine the association between withdrawal of SSRI, SNRI, and NaSSa and the risk reduction of recurrent falls.

\section{CONCLUSION}

In conclusion, the use of SSRI, SNRI, and NaSSa was associated with an increased risk of recurrent falls. Additionally, serious attention should be paid to women and elderly patients administered SSRI, SNRI, and NaSSa to avoid recurrent falls. Further studies are warranted to validate these results using a large sample size.

Acknowledgments We greatly appreciate all nurses working in Tokyo Women's University, Medical Center East for their precise recordings of fall events.

Conflict of Interest The authors declare no conflict of interest.

\section{REFERENCES}

1) Basic D, Hartwell TJ. Falls in hospital and new placement in a nursing home among older people hospitalized with acute illness. Clin. Interv. Aging, 10, 1637-1643 (2015).

2) Sterke CS, Ziere G, van Beeck EF, Looman CW, van der Cammen TJ. Dose-response relationship between selective serotonin reuptake inhibitors and injurious falls: a study in nursing home residents with dementia. Br. J. Clin. Pharmacol., 73, 812-820 (2012).
3) Avidan AY, Fries BE, James ML, Szafara KL, Wright GT, Chervin $\mathrm{RD}$. Insomnia and hypnotic use, recorded in the minimum data set, as predictors of falls and hip fractures in Michigan nursing homes. J. Am. Geriatr. Soc., 53, 955-962 (2005).

4) Hien TT, Cumming RG, Cameron ID, Chen JS, Lord SR, March LM, Schwarz J, Le Couteur DG, Sambrook PN. Atypical antipsychotic medications and risk of falls in residents of aged care facilities. J. Am. Geriatr. Soc., 53, 1290-1295 (2005).

5) Vlaeyen E, Coussement J, Leysens G, Van der Elst E, Delbaere K, Cambier D, Denhaerynck K, Goemaere S, Wertelaers A, Dobbels F, Dejaeger E, Milisen K. Characteristics and effectiveness of fall prevention programs in nursing homes: a systematic review and meta-analysis of randomized controlled trials. J. Am. Geriatr. Soc., 63, 211-221 (2015).

6) Mazur K, Wilczynski K, Szewieczek J. Geriatric falls in the context of a hospital fall prevention program: delirium, low body mass index, and other risk factors. Clin. Interv. Aging, 11, 1253-1261 (2016).

7) Ylitalo KR, Karvonen-Gutierrez CA. Body mass index, falls, and injurious falls among U.S. adults: Findings from the 2014 Behavioral Risk Factor Surveillance System. Prev. Med., 91, 217-223 (2016).

8) van der Velde N, Stricker BH, Pols HA, van der Cammen TJ. Risk of falls after withdrawal of fall-risk-increasing drugs: a prospective cohort study. Br. J. Clin. Pharmacol., 63, 232-237 (2007).

9) Hempel S, Newberry S, Wang Z, Booth M, Shanman R, Johnsen B, Shier V, Saliba D, Spector WD, Ganz DA. Hospital fall prevention: a systematic review of implementation, components, adherence, and effectiveness. J. Am. Geriatr. Soc., 61, 483-494 (2013).

10) Hartikainen $S$, Lonnroos $E$, Louhivuori $K$. Medication as a risk factor for falls: critical systematic review. J. Gerontol. A Biol. Sci. Med. Sci., 62, 1172-1181 (2007).

11) Marcum ZA, Perera S, Newman AB, Thorpe JM, Switzer GE, Gray SL, Simonsick EM, Shorr RI, Bauer DC, Castle NG, Studenski SA, Hanlon JT. Antihypertensive use and recurrent falls in communitydwelling older adults: findings from the health $\mathrm{ABC}$ study. $J$. Gerontol. A Biol. Sci. Med. Sci., 70, 1562-1568 (2015).

12) Ensrud KE, Blackwell TL, Mangione CM, Bowman PJ, Whooley MA, Bauer DC, Schwartz AV, Hanlon JT, Nevitt MC. Central nervous system-active medications and risk for falls in older women. $J$. Am. Geriatr. Soc., 50, 1629-1637 (2002).

13) Matsuo S, Imai E, Horio M, Yasuda Y, Tomita K, Nitta K, Yamagata $\mathrm{K}$, Tomino $\mathrm{Y}$, Yokoyama $\mathrm{H}$, Hishida A. Revised equations for estimated GFR from serum creatinine in Japan. Am. J. Kidney Dis., 53, 982-992 (2009).

14) The prevention of falls in later life. A report of the Kellogg International Work Group on the prevention of falls by the elderly. Dan. Med. Bull., 34 (Suppl. 4), 1-24 (1987).

15) Tariq H, Kloseck M, Crilly RG, Gutmanis I, Gibson M. An exploration of risk for recurrent falls in two geriatric care settings. $B M C$ Geriatr., 13, 106 (2013).

16) de Jong MR, Van der Elst M, Hartholt KA. Drug-related falls in older patients: implicated drugs, consequences, and possible prevention strategies. Ther Adv. Drug Saf., 4, 147-154 (2013).

17) Gale CR, Cooper C, Aihie Sayer A. Prevalence and risk factors for falls in older men and women: the english longitudinal study of ageing. Age Ageing, 45, 789-794 (2016)

18) Kallin K, Lundin-Olsson L, Jensen J, Nyberg L, Gustafson Y. Predisposing and precipitating factors for falls among older people in residential care. Public Health, 116, 263-271 (2002).

19) Coupland C, Dhiman P, Morriss R, Arthur A, Barton G, HippisleyCox J. Antidepressant use and risk of adverse outcomes in older people: population based cohort study. $B M J, 343$ (aug02 1), d4551 (2011).

20) Gribbin J, Hubbard R, Gladman J, Smith C, Lewis S. Serotoninnorepinephrine reuptake inhibitor antidepressants and the risk of falls in older people: case-control and case-series analysis of a large 
U.K. primary care database. Drugs Aging, 28, 895-902 (2011).

21) Naples JG, Kotlarczyk MP, Perera S, Greenspan SL, Hanlon JT. Non-tricyclic and non-selective serotonin reuptake inhibitor antidepressants and recurrent falls in frail older women. Am. J. Geriatr. Psychiatry, 24, 1221-1227 (2016).

22) Moura C, Bernatsky S, Abrahamowicz M, Papaioannou A, Bessette L, Adachi J, Goltzman D, Prior J, Kreiger N, Towheed T, Leslie WD, Kaiser S, Ioannidis G, Pickard L, Fraser LA, Rahme E. Antidepressant use and 10-year incident fracture risk: the populationbased Canadian Multicentre Osteoporosis Study (CaMoS). Osteoporos. Int., 25, 1473-1481 (2014).

23) Lanteigne A, Sheu YH, Sturmer T, Pate V, Azrael D, Swanson SA, Miller M. Serotonin-norepinephrine reuptake inhibitor and selective serotonin reuptake inhibitor use and risk of fractures: a newuser cohort study among U.S. adults aged 50 years and older. CNS Drugs, 29, 245-252 (2015).

24) Vestergaard P, Rejnmark L, Mosekilde L. Selective serotonin reuptake inhibitors and other antidepressants and risk of fracture. Calcif. Tissue Int., 82, 92-101 (2008).

25) Wang CY, Fu SH, Wang CL, Chen PJ, Wu FL, Hsiao FY. Serotonergic antidepressant use and the risk of fracture: a population-based nested case-control study. Osteoporos. Int., 27, 57-63 (2016).

26) Scheffer AC, Schuurmans MJ, van Dijk N, van der Hooft T, de Rooij SE. Fear of falling: measurement strategy, prevalence, risk factors and consequences among older persons. Age Ageing, 37, 19-24 (2008).

27) Khan A, Brodhead AE, Schwartz KA, Kolts RL, Brown WA. Sex differences in antidepressant response in recent antidepressant clinical trials. J. Clin. Psychopharmacol., 25, 318-324 (2005).

28) Lobo ED, Quinlan T, O’Brien L, Knadler MP, Heathman M. Population pharmacokinetics of orally administered duloxetine in patients: implications for dosing recommendation. Clin. Pharmacokinet., 48, 189-197 (2009).

29) Skinner MH, Kuan HY, Skerjanec A, Seger ME, Heathman M, O'Brien L, Reddy S, Knadler MP. Effect of age on the pharmacokinetics of duloxetine in women. Br. J. Clin. Pharmacol., 57, 54-61 (2004).

30) Polasek TM, Patel F, Jensen BP, Sorich MJ, Wiese MD, Doogue MP. Predicted metabolic drug clearance with increasing adult age. Br. J. Clin. Pharmacol., 75, 1019-1028 (2013).

31) Hayashida I, Tanimoto Y, Takahashi Y, Kusabiraki T, Tamaki J. Correlation between muscle strength and muscle mass, and their association with walking speed, in community-dwelling elderly Japanese individuals. PLOS ONE, 9, e111810 (2014).

32) Gleason CE, Gangnon RE, Fischer BL, Mahoney JE. Increased risk for falling associated with subtle cognitive impairment: secondary analysis of a randomized clinical trial. Dement. Geriatr. Cogn. Disord., 27, 557-563 (2009). 